\title{
AN INTEGRATED, MULTISTUDY ANALYSIS OF THE SAFETY OF ANN ARBOR STRAIN LIVE ATTENUATED INFLUENZA VACCINE IN CHILDREN AGED 2-17 YEARS
}

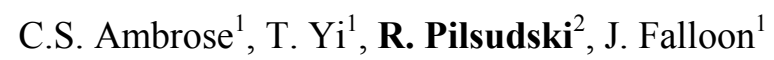 \\ ${ }^{1}$ MedImmune, LLC, Gaithersburg, MD, USA, ${ }^{2}$ AstraZeneca, Paris, France
}

Background and aims: A trivalent, intranasal Ann Arbor strain live attenuated influenza vaccine (AALAIV) is approved in several countries for use in eligible children aged $\geq 2$ years. This analysis assesses the safety of AA-LAIV in children aged 2-17 years.

Methods: Randomized, controlled studies of AA-LAIV were evaluated. Solicited reactogenicity events (REs) and unsolicited adverse events (AEs) were summarized for days 0-10 postvaccination. Serious AEs (SAEs) were summarized through 42 and 180 days postvaccination. AEs and SAEs were summarized by MedDRA system organ class and preferred term.

Results: 4245 and 10,693 children received AA-LAIV in year 1 of 6 TIV-controlled and 14 placebocontrolled studies, respectively; 3212 children were revaccinated in year 2 of 4 placebo-controlled studies. REs increased following AA-LAIV versus placebo were runny/stuffy nose $(+7 \%)$, headache $(+7 \%)$, and lethargy $(+2 \%)$ after dose 1 , and decreased appetite $(+4 \%)$ after year 2 revaccination. Compared with TIV, only runny/stuffy nose was increased (dose $1,+12 \%$; dose $2,+4 \%$ ). Compared with initial vaccination, AALAIV reactogenicity was lower after revaccination. AEs increased with AA-LAIV in some comparisons were headache, nasal congestion/rhinorrhea, rhinitis, and pyrexia; ear pain and lower respiratory illness were decreased. There was no evidence of an increase in lower respiratory illness, wheezing illness, or any potential vaccine-related SAE in AA-LAIV recipients.

Conclusions: This integrated analysis of the safety of AA-LAIV supports the safety of AA-LAIV in eligible children aged 2-17 years and provides a consensus assessment of the expected events postvaccination. 